\title{
ЧИСЛЕННОЕ МОДЕЛИРОВАНИЕ ДИНАМИКИ ИОНОСФЕРНЫХ ПРОЦЕССОВ В ЕСТЕСТВЕННЫХ УСЛОВИЯХ И УСЛОВИЯХ ТЕХНОГЕННОГО ВОЗДЕЙСТВИЯ 1
}

\section{NUMERICAL MODELING OF THE DYNAMICS OF IONOSPHERIC PROCESSES IN NATURAL AND MAN-MADE CONDITIONS}

E. Zubkov

K. Bogomolov Yu. Dedkov S. Shilova

Summary. A method for numerical modeling of processes in near-Earth space plasma under natural and anthropogenic effects is presented.

The method is implemented in a non-stationary model of the ionosphereplasmosphere system, which allows one to calculate the distribution of characteristics of low-energy plasma along geomagnetic field lines of arbitrary size, taking into account ambipolar diffusion, photochemical processes, and interaction with a horizontal atmospheric wind.

The mathematical model was used to study the physicochemical processes in the ionospheric plasma, the processes of the dynamic and chemical effects on this plasma medium.

The results of modeling variations in the parameters of ions and electrons in the mid-latitude ionosphere and magnetosphere are presented.

Keywords: Ionosphere, plasmasphere, mathematical modeling, numerical simulating, technogenic impact, dipole geomagnetic field.
Зубков Евгений Вячеславович

Аспирант, ФГАОУ ВО «Балтийский федеральный университет имени И. Канта» evgenyzubkov@mail.ru

Богомолов Константин Юрьевич Аспирант, ФГАОУ ВО «Балтийский федеральный университет имени И. Канта»

kst44@mail.ru

Дедков Юрий Викторович

Аспирант, ФГАОУ ВО «Балтийский федеральный университет имени И. Канта» yuri.dedkov@gmail.com

Шилова София Олеговна

Аспирант, ФГАОУ ВО «Балтийский федеральный университет имени И. Канта» ya.shelby95@ya.ru

Аннотация. Представлен метод численного моделирования процессов в околоземной космической плазме в естественных условиях и условиях антропогенного воздействия.

Метод реализован в нестационарной модели системы ионосфера-плазмосфера, которая позволяет рассчитывать распределения характеристик низкоэнергичной плазмы вдоль геомагнитных силовых линий произвольного размера с учетом амбиполярной диффузии, фотохимических процессов, взаимодействия с горизонтальным атмосферным ветром.

Математическая модель использовалась для исследования физико-химических процессов в ионосферной плазме, процессов динамического и химического воздействия на эту плазменную среду.

Приведены результаты моделирования вариаций параметров ионов и электронов в среднеширотной ионосфере и магнитосфере.

Ключевые слова: ионосфера, плазмосфера, математическое моделирование, численное моделирование, техногенные воздействия, дипольное геомагнитное поле. 


\section{Ввемение}

B исследовании ионосферных процессов и явлений важную роль играет разработка физико-математических моделей низкоэнергичной околоземной космической плазмы [1-3].

Исследование различных динамических процессов (транспорт веществ, распространение радиоволн, МГДволн и др.) в магнитосфере и ионосфере Земли имеет следующие особенности:

- большая (экспоненциальная по плотности и давлению) неоднородность атмосферы Земли;

- длительные времена подлежащих моделированию процессов эволюции плазменных образований под совместным действием поля гравитации и магнитного поля Земли;

- сильно различающиеся доли внутренней и кинетической энергии плазмы в возмущенной области;

- сложный комплекс физико-химических процессOB.

Ионосферно-магнитосферная плазма представляет собой весьма сложную для описания среду прежде всего из-за того, что в нижней своей части, ионосферной, это - слабоионизованная плазма со степенью ионизации 10-2-10-3 (область главного ионосферного максимума), а на больших высотах, в магнитосферной части, она переходит в полностью ионизованную плазмy.

Особый интерес представляют исследования околоземной среды в условиях искусственного воздействия на нее.

В качестве таких техногенных воздействий рассматриваются выбросы сгустков легкоионизируемого вещества, плазменных струй, возмущения типа мощной солнечной вспышки (радиоволны), инжекция пучков химически активных нейтральных и заряженных частиц [4-6].

В связи с этим представляются актуальными задачи исследования околоземной плазменной среды с использованием математических моделей, которые учитывают различные типы антропогенных воздействий.

\section{Описание модели \\ и численных алгоритмов}

Магнитогидродинамическое описание ионосферной плазмы наиболее естественным образом осуществляется в системе координат, связанной с геомагнитным полем.
Выбором дипольной системы координат сведем физическую задачу трехмерного движения плазмы к двумерной математической задаче в переменных $s$ (координата вдоль силовой линии) и $t$ (время).

Пространственную картину распределения плазмы можно получить, если учесть ее электрический дрейф. Состав плазмы определим наличием электронов, ионов $\mathrm{O}^{+}\left({ }^{4} \mathrm{~S}\right), \mathrm{H}^{+}, \mathrm{NO}^{+}, \mathrm{O}_{2}^{+}, \mathrm{N}_{2}^{+}$и нейтральных частиц $\mathrm{O}_{2}, \mathrm{~N}_{2}, \mathrm{O}, \mathrm{H}, \mathrm{H}_{2} \mathrm{O}$, концентрации которых обозначим через $n_{e}, n_{i}(i=\overline{1,5}), N_{n}(n=\overline{1,5})$ соответственно.

Математическая модель основана на численном решении системы уравнений гидродинамики частично ионизированной плазмы для ионов и электронов.

Запишем сначала уравнения для ионов $\mathrm{O}^{+}\left({ }^{4} S\right)$ и $\mathrm{H}^{+}$, являющихся основными на высотах $h>200$ км. Уравнение непрерывности будут иметь следующий вид:

$$
\begin{aligned}
& \frac{\partial n_{1}}{\partial t}+\frac{1}{A(s)} \frac{\partial}{\partial s}\left(A n_{1} u_{1}\right)=q_{1}+\gamma_{5} N_{3} n_{2}- \\
& -\left(\gamma_{1} N_{2}+\gamma_{2} N_{1}+\gamma_{3} N_{4}+\gamma_{4} N_{5}\right) n_{1} ; \\
& \frac{\partial n_{2}}{\partial t}+\frac{1}{A(s)} \frac{\partial}{\partial s}\left(A n_{2} u_{2}\right)=\gamma_{3} N_{4} n_{1}-\gamma_{5} N_{3} n_{2} .
\end{aligned}
$$

Уравнения движения для ионов:

$$
\begin{aligned}
& u_{j}=\frac{1}{R_{j}+s_{j i}}\left[m_{j} g \sin I+s_{j i} u_{i}+R_{j} V_{n x} \cos I-\right. \\
& \left.-\frac{k}{n_{e}} \frac{\partial\left(n_{e} T_{e}\right)}{\partial s}-\frac{k}{n_{j}} \frac{\partial\left(n_{j} T_{j}\right)}{\partial s}\right]
\end{aligned}
$$

Уравнения теплового баланса для электронов и ионов запишем в виде

$$
\begin{aligned}
& \frac{3}{2} k n_{e} \frac{\partial T_{e}}{\partial t}=\frac{1}{A} \frac{\partial}{\partial s}\left(A \lambda_{e} \frac{\partial T_{e}}{\partial s}\right)+3 n_{e} m_{e} k \sum_{i} \frac{v_{e i}^{*}}{m_{i}}\left(T_{i}-\right. \\
& \left.-T_{e}\right)+Q_{e}^{c m}-\sum_{n} L_{e n}, \\
& \frac{3}{2} k n_{1} \frac{\partial T_{1}}{\partial t}=\frac{1}{A} \frac{\partial}{\partial s}\left(A \lambda_{1} \frac{\partial T_{1}}{\partial s}\right)+3 n_{1} v_{1 e}^{*} k\left(T_{e}-T_{1}\right)+ \\
& +\frac{3 m_{1} n_{1}}{m_{1}+n_{1}} v_{12}^{*} k\left(T_{2}-T_{1}\right)++\sum_{n} \frac{3 m_{1} n_{1}}{m_{1}+m_{n}} v_{1 n} k\left(T_{n}-T_{1}\right)+ \\
& \frac{3}{2} \gamma_{5} N_{3} n_{2} k\left(T_{n}-T_{1}\right)-\frac{1}{2} \frac{\gamma_{3} N_{4} n_{4} k T_{1}}{1+16 T_{n} / T_{1}}, \\
& \frac{3}{2} k n_{2} \frac{\partial T_{2}}{\partial t}=\frac{1}{A} \frac{\partial}{\partial s}\left(A \lambda_{2} \frac{\partial T_{2}}{\partial s}\right)+3 n_{2} v_{2 e}^{*} k\left(T_{e}-T_{2}\right)+
\end{aligned}
$$




$$
\begin{aligned}
& +\frac{3 m_{2} n_{2}}{m_{1}+n_{2}} v_{21}^{*} k\left(T_{1}-T_{2}\right)+\sum_{n} \frac{3 m_{2} n_{2}}{m_{2}+m_{n}} v_{2 n} k\left(T_{n}-T_{2}\right)+ \\
& +\gamma_{3} N_{4} n_{1} k\left(2 T_{n}-\frac{3}{2} T_{2}\right)-\frac{1}{2} \gamma_{5} N_{3} n_{2} k T_{2}
\end{aligned}
$$

В качестве граничных условий в основания силовой трубки задаются равенства $T_{e}=T_{i}=T_{n}$.

Будем считать выполненным условие квазинейтральности плазмы

$$
n_{e}=\sum_{k=1}^{5} n_{k} \text {. }
$$

Здесь индекс $е$ относится к электронам, индексы $i=$ 1,2 и $j=1,2-$ к ионам, индекс $n-$ к нейтральным частицам; $A$ - расходимость силовых линий геомагнитного поля; $I$ - магнитное наклонение; $R_{j}-$ коэффициент силы трения между ионами $j$-го сорта и нейтральными частицами; $S_{j i}$ - коэффициент силы трения между ионами $i$-го сорта и $j$-го сортов; $V_{n x}-$ меридиональная составляющая скорости нейтрального ветра; $q_{1}-$ скорость ионизации атомарного кислорода солнечным ультрафиолетовым (УФ) излучением; $m_{j}, u_{j}, T_{j}-$ масса, скорость, температура частиц $j$-го сорта соответственно; $k$ - постоянная Больцмана; $g$ - ускорение силы тяжести; $\gamma_{1}, \gamma_{2}, \gamma_{3}, \gamma_{4}, \gamma_{5}$ - коэффициенты химических реакций $[5,6] ; \lambda_{i}, \lambda_{e}-$ коэффициенты теплопроводности ионного и электронного газов; $v_{i n}$ и $v_{i j}^{*}-$ частоты столкновений ионов с нейтральными частицами и друг с другом; $v_{i e}^{*}-$ частоты кулоновских столкновений ионов с тепловыми электронами; $Q_{e}^{c m}-$ скорость нагрева электронного газа сверхтепловыми электронами; $L_{e n}-$ скорость теплообмена электронов с нейтральными частицами.

Конкретные выражения для $L_{e n}, \lambda_{e}, \lambda_{i}, \lambda_{i n}, v_{i j}^{*}$ имеются в работе [6]. Скорость нагрева тепловых электронов $Q_{e}^{c m}$, входящая в уравнение теплового баланса, вычисляется по формуле:

$$
\begin{aligned}
& Q_{e}^{c m}=\int_{0}^{E_{M}} E Q(E) d E+4 \pi k N_{e}\left[\Phi_{0}\left(E_{M}\right)+\right. \\
& \left.+\int_{E_{M}}^{\infty} \Phi_{0}(E) \frac{d E}{E}\right],
\end{aligned}
$$

где $E$ - энергия электрона;

$E_{M}$ - граничная энергия, начиная с которой максвелловское распределение электронов начинает существенно искажаться;

$Q(E)$ - скорость образования фотоэлектронов с энергией от $E$ до $E+d E$ при ионизации солнечным УФ-излучением;

$$
k=2.6 \cdot 10^{-12} э в \cdot \mathrm{CM}^{-2}
$$

$\Phi_{0}(E)$ - изотропная часть потока сверхтепловых электронов.

Потоки сверхтепловых электронов рассчитываются путем решения кинетического уравнения, взятого в квазистационарном дрейфовом приближении, согласно работе [7].

Меридиональная составляющая скорости нейтрального ветра $V_{n x}$ входящая в уравнение движения (3), находится из решения системы уравнений:

$$
\begin{aligned}
& \frac{\partial V_{n x}}{\partial t}=\frac{\xi}{\sin ^{2} I} \frac{\partial^{2} V_{n x}}{\partial s^{2}}-\frac{1}{\rho_{n}} \sum_{j=1}^{5} n_{j} R_{j}\left(V_{n x}-V_{j} \cos I\right)+ \\
& +2 \Omega \sin \varphi V_{n y}-\frac{1}{\rho_{n}} \frac{\partial P_{n}}{\partial x} \\
& \frac{\partial V_{n y}}{\partial t}=\frac{\xi}{\sin ^{2} I} \frac{\partial^{2} V_{n y}}{\partial s^{2}}-\frac{1}{\rho_{n}} \sum_{j=1}^{5} n_{j} R_{j} V_{n y}- \\
& -2 \Omega \sin \varphi V_{n x}-\frac{1}{\rho_{n}} \frac{\partial P_{n}}{\partial y}
\end{aligned}
$$

Здесь $V_{n y}$ - зональная составляющая скорости зонального ветра;

$\xi$ - кинематическая вязкость нейтрального газа;

$\rho_{n}$ - плотность нейтрального газа;

$\Omega$-угловая скорость вращения Земли;

$\varphi$ - географическая широта.

Меридиональная и зональная компоненты градиента давления нейтрального газа

$$
\frac{\partial P_{n}}{\partial x} \text { и } \frac{\partial P_{n}}{\partial y}
$$

рассчитывается согласно работе [1]. На нижней границе $s=h_{0}$ считаем, что $V_{n x}=V_{n y}=0$, на верхней границе $s=800$ км задаем нулевые градиенты скоростей.

Высотно-временное распределение $k$ - $\breve{u}$ инжектируемой нейтральной компоненты с учетом процессов переноса будем получать как решение уравнения непрерывности

$$
\frac{\partial N_{k}}{\partial t}+\frac{\partial\left(N_{k} V_{k}\right)}{\partial s}=Q_{k}-\alpha_{k} N_{k},
$$

где $V_{л}$ - молекулярная скорость движения частиц $k$-й компоненты, $Q_{k}$ и $\alpha_{k}-$ члены, учитывающие образование и потери частиц в фотохимических реакциях.

Численное решение уравнения (10) проводилось согласно работе [6].

Нестационарные уравнения фотохимического баланса для молекулярных ионов $\mathrm{O}_{2}^{+}, \mathrm{NO}^{+}, \mathrm{N}_{2}^{+}$записываются в виде 

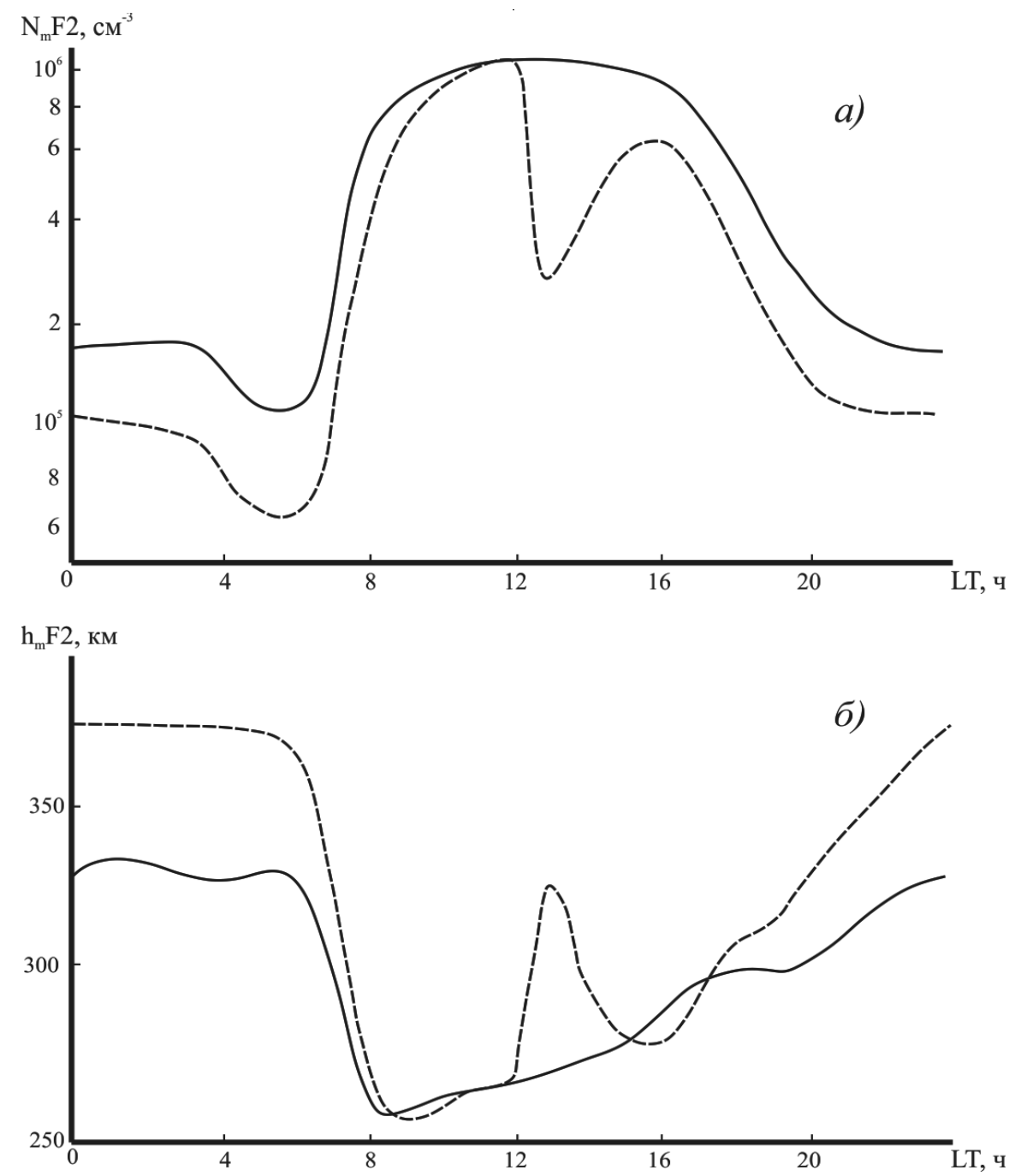

Рис. 1. Суточные вариации максимального значения электронной концентрации $N_{m} F 2$ в области $F 2$ ионосферы (а) и высоты $h_{m} F 2$, на которой находится это максимальное значение (б) (невозмущенные условия - сплошная кривая, с учетом инжектируемой воды - пунктирная кривая).

$$
\frac{\partial n_{i}}{\partial t}=Q_{i}-\alpha_{i} n_{i}, \quad i=3,4,5
$$

Численное решение системы уравнений модели проводилось вдоль геомагнитной силовой линии с использованием метода конечных разностей.

Линеаризация разностных уравнений проводилась с использованием значений неизвестных функций, взятых с предыдущего временного слоя, с последующими итерациями по нелинейности и связанности уравнений.

\section{Результаты численных расчетов}

Рассматривалась геомагнитная силовая трубка с параметром Мак-Илвайна $L=3$, основание которой располагалось на высоте $h_{0}=125 \mathrm{~km}$.
Расчеты проводились для средней геомагнитной $\left(k_{p}=3\right)$ и солнечной $\left(F_{10.7}=140\right)$ активности, зимних условий (36 день от начала года).

В данных расчетах использовалась модель нейтральной атмосферы MSIS [8].

В качестве начальных условий для уравнений физико-математической модели сначала задавались произвольные в допустимых пределах начальные распределения вдоль силовой трубки, от которых проводилось интегрирование до выхода решений на установившейся периодический, суточный режим, соответствующий невозмущенным условиям.

Затем в момент времени $L T=12$ ч включалась инжектируемая компонента $H_{2} 0$, которая учитывалась при 


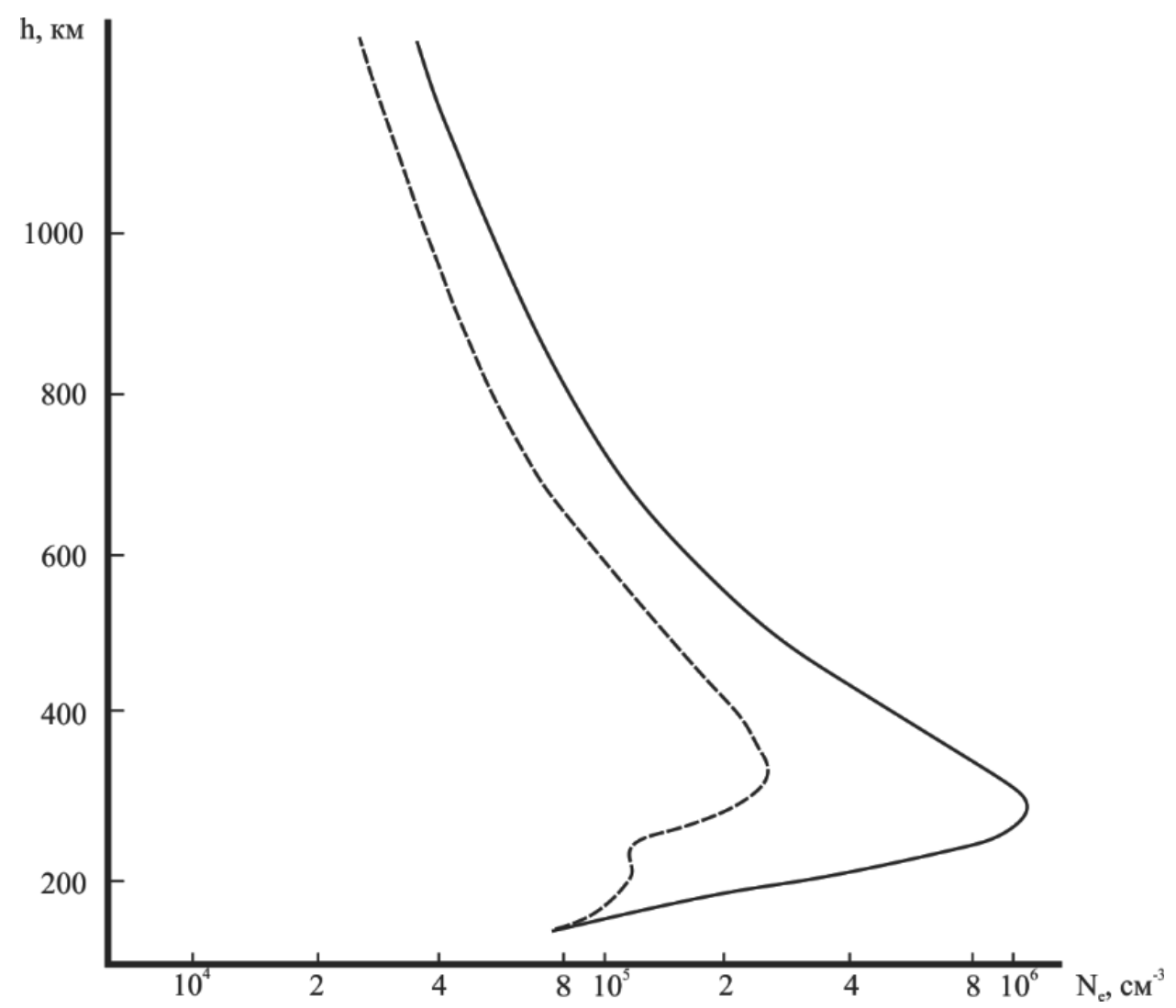

Рис. 2. Высотные профили электронной концентрации для 13 час. Обозначения см. на рис. 1.

решении уравнения диффузии для атомарного водорода.

В качестве начальных условий для $\mathrm{H}_{2} \mathrm{O}$ задавалось барометрическое распределение.

Уравнение диффузии для $H$ и $\mathrm{H}_{2} \mathrm{O}$ решались в ограниченной области высот $125 \kappa m \leq s \leq 800 \kappa м$.

На рис. 1 приведены рассчитанные суточные вариации максимального значения электронной концентрации $N_{m} F 2$ в области $F 2$ ионосферы и высоты $h_{m} F 2$, на которой находится это максимальное значение, для невозмущенных условий (сплошная кривая) и с учетом инжектируемой воды (пунктирная кривая).

Выброс воды, начатый в полдень, приводит к резкому уменьшению $N_{m} F 2$ (примерно в три раза) к моменту $L T=13$ ч что можно объяснить действием $\left[\mathrm{H}_{2} \mathrm{O}\right]$ как источника потерь ионов $\mathrm{O}^{+}$. Очевидно, что амплитуда выброса зависит от количества инжектируемой воды. На этом же временном интервале $12 \leq L T \leq 13$ происходит рост $h_{m} F 2$ - высоты максимума $n_{e}$. Образование ионосферной «дыры», сопровождающееся ростом $h_{m} F 2$, подтверждается рис. 2, на котором представлены не- возмущенный высотный профиль $N_{e}$ (сплошная кривая) и профиль для $L T=13$ ч (пунктирная кривая).

В дальнейшем в промежуток времени $13 \leq L T \leq 16$ происходит затягивание ионосферной «дыры» (увеличение $\left[n_{e}\right]$, рис. 1, а). Это увеличение $\left[n_{e}\right]$ можно объяснить уменьшением $\left[\mathrm{H}_{2} \mathrm{O}\right]$. Однако более существенным фактором является изменение потока ионизованных частиц. Как известно, для нормальных условий поток заряженных частиц направлен днем из $F 2$ области в магнитосферу. Образование же ионосферной «дыры» привело к изменению направления потока на противоположное. Данный эффект иллюстрирует рис. 3.

Для нормальных дневных условий поток частиц выше F2 области направлен вверх, то есть положителен, и составляет $2 \cdot 10^{8} \mathrm{~cm}^{-2} \mathrm{c}^{-1}$. Для возмущенных условий такой же примерно по величине поток при $L T=13$ ч направлен вниз (то есть отрицателен). На рис. 4 показана суточная вариация невозмущенного (сплошная кривая) и с учетом выброса воды (пунктирная кривая) потока плазмы на высоте $h=840$ км и наличие во время образования ионосферной дыры отрицательного потока, который приводит в дальнейшем к ее заполнению. Высотные профили температуры электронов для спокойных и воз- 


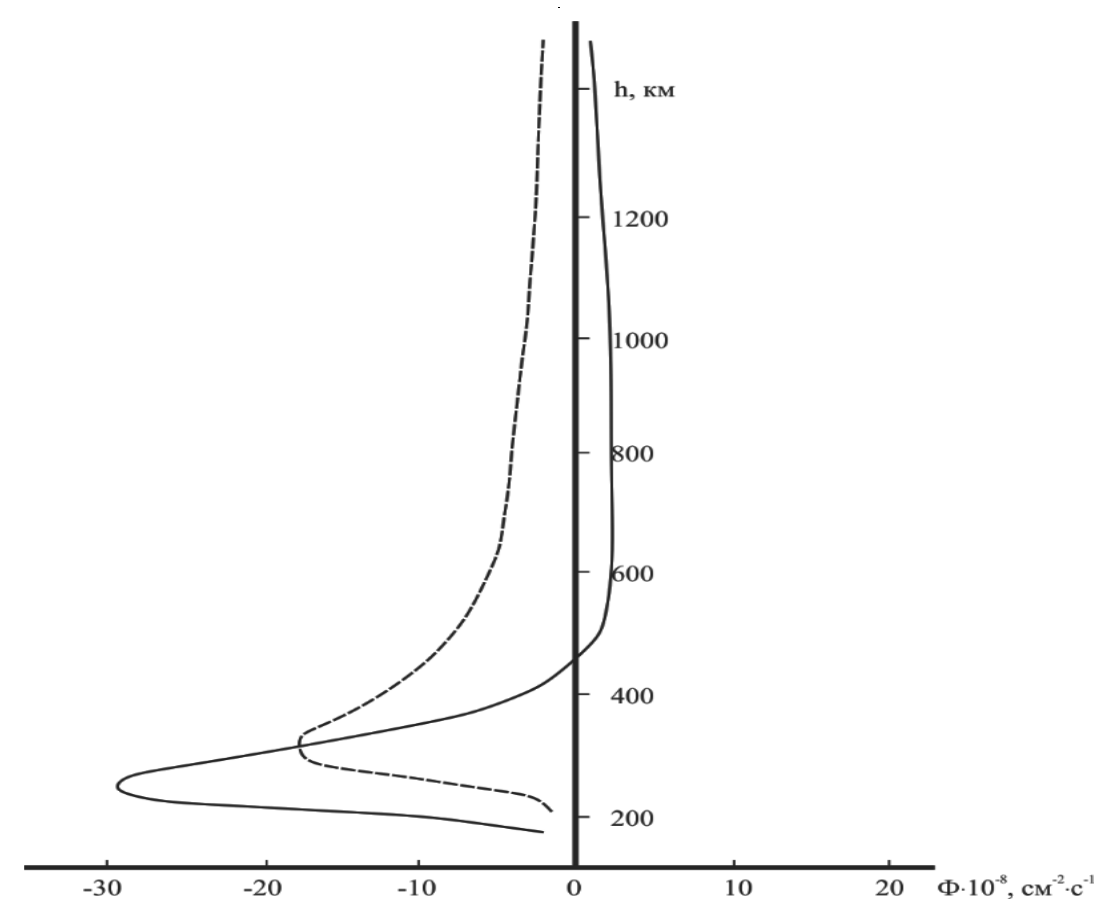

Рис. 3. Высотные профили ионных потоков в северном полушарии для 13 ч. Обозначения см. на рис. 1.

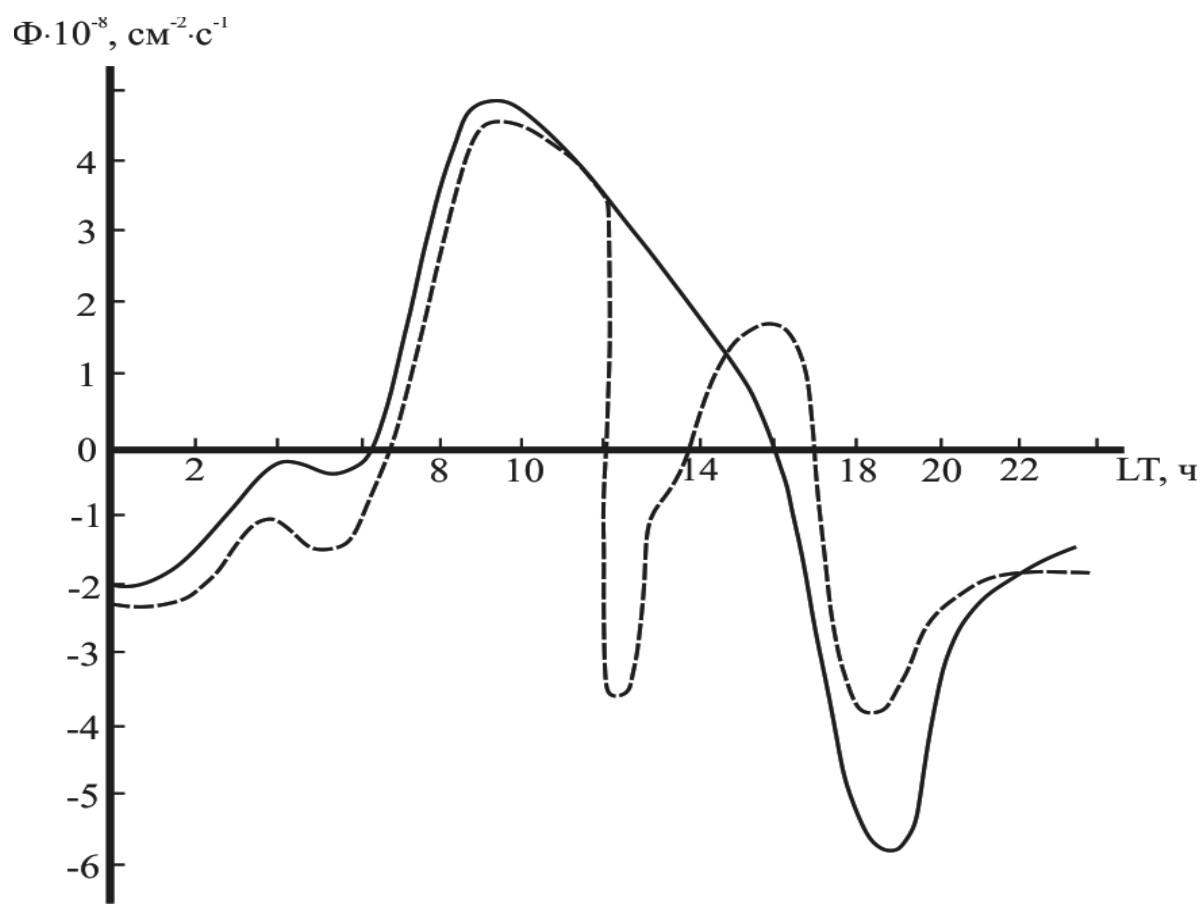

Рис. 4. Временные вариации суммарного ионного потока на высоте 840 км. Обозначения см. на рис. 1.

мущенных условий и $L T=13$ ч представлены на рис. 5 . Уменьшение электронной концентрации до значений $N_{m} F 2=2 \cdot 10^{5} \mathrm{~cm}^{-3}$ приводит к росту электронной температуры в $F 2$ области до $3500^{\circ} \mathrm{K}$.

\section{Зак^ючение}

Представленная в работе математическая модель позволяет на основе численного решения уравнений непрерывности, движения и теплового баланса много- 


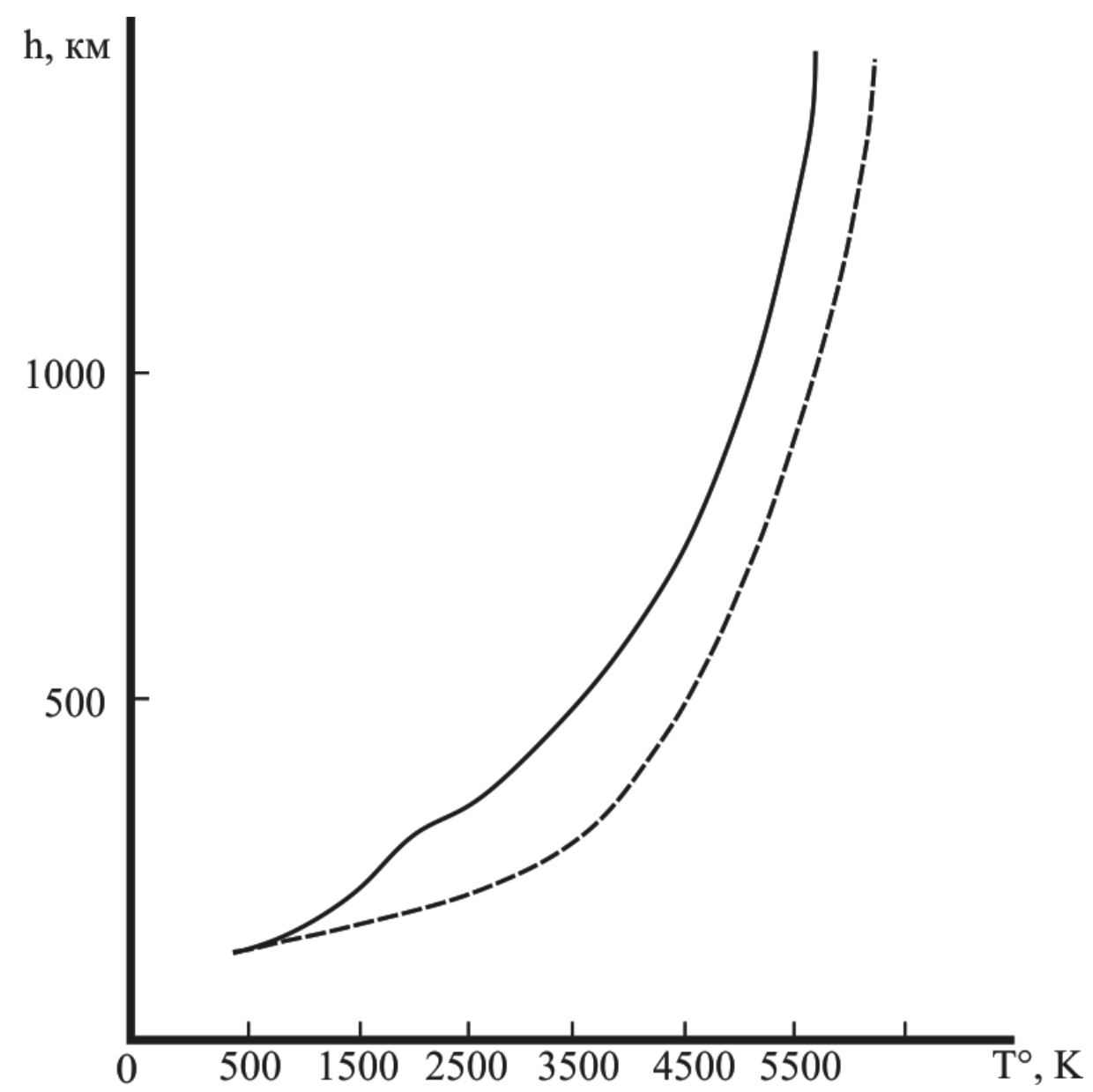

Рис. 5. Высотный профиль электронной температуры для 13 LT. Обозначения см. на рис. 1.

компонентной плазмы, записанных для геомагнитных силовых трубок, исследовать процессы модификации ионосферы при техногенных воздействиях.

Показано, что при выпусках на высотах $F 2$ - слоя ионосферы некоторых химически активных газов происходит перестройка электронной компоненты плазмы, образования «ионосферных дыр», разогрев плазменных компонент, обмен между плазмосферой и ионосферой потоками заряженных частиц.

Амплитуды изменения параметров ионосферной плазмы зависят как от мощности самого воздействия, так и от расстояния до центра возмущения.

\section{ЛИТЕРАТУРА}

1. Латышев К.С., Зинин Л. В., Ишанов С. А. Математическое моделирование околоземной космической плазмы // Энциклопедия низкотемпературной плазмы. - 2008. - Т. 7, Ч. 3.- С. 337-349.

2. Тащилин А.В., Романова Е. Б. Численное моделирование диффузии ионосферной плазмы в дипольном геомагнитном поле при наличии поперечного дрейфа // Математическое моделирование.-2013. - Т. 25, № 1.- С. 3-17.

3. Е.В. Зубков, П. М. Каратаева, К. Ю. Богомолов, Д. А. Савкин, В. Н. Худенко. Численное моделирование ионосферного распространения коротких радиоволн // Актуальные проблемы прикладной математики, информатики и механики. Сборник трудов Международной научно-технической конференции Воронеж, 11-13 ноября 2019 г. С. 821-826.

4. Ступицкий Е.Л., Холодов А.С. Физические исследования и математическое моделирование крупномасштабных геофизических экспериментов: Монография // Издательский Дом Интеллект. - 2018.

5. Ишанов С.А., Мацула П. В. Вычислительный эксперимент при моделировании динамики антропогенных возмущений ионосферно-магнитосферной плазмы // Математическое моделирование.— 2012.— - Т. 24, № 6.—C. 128-136. 
6. Ишанов С.А., Леванов Е. И., Медведев В. В., Залеская В. А. Магнитосферно-ионосферные изменения, вызванные полетами космических аппаратов // Инженерно-физический журнал. Национальная Академия наук Беларуси. - 2006. - Т. 79, № 6. - С. 11-15.

7. Кринберг Н.А., Тащилин А. В. Ионосфера и плазмосфера.—- М.: Наука, 1984.

8. Hedin A.E. MSIS-86 thermospheric model // J. Geophys. Res. — 1987.— Vol. 92, № 5. — P. 4649-4662.

( ) Зубков Евгений Вячеславович ( evgenyzubkov@mail.ru ), Богомолов Константин Юрьевич ( kst44@mail.ru ), Дедков Юрий Викторович ( yuri.dedkov@gmail.com ), Шилова София Олеговна ( ya.shelby95@ya.ru ).

Журнал «Современная наука: актуальные проблемы теории и практики»

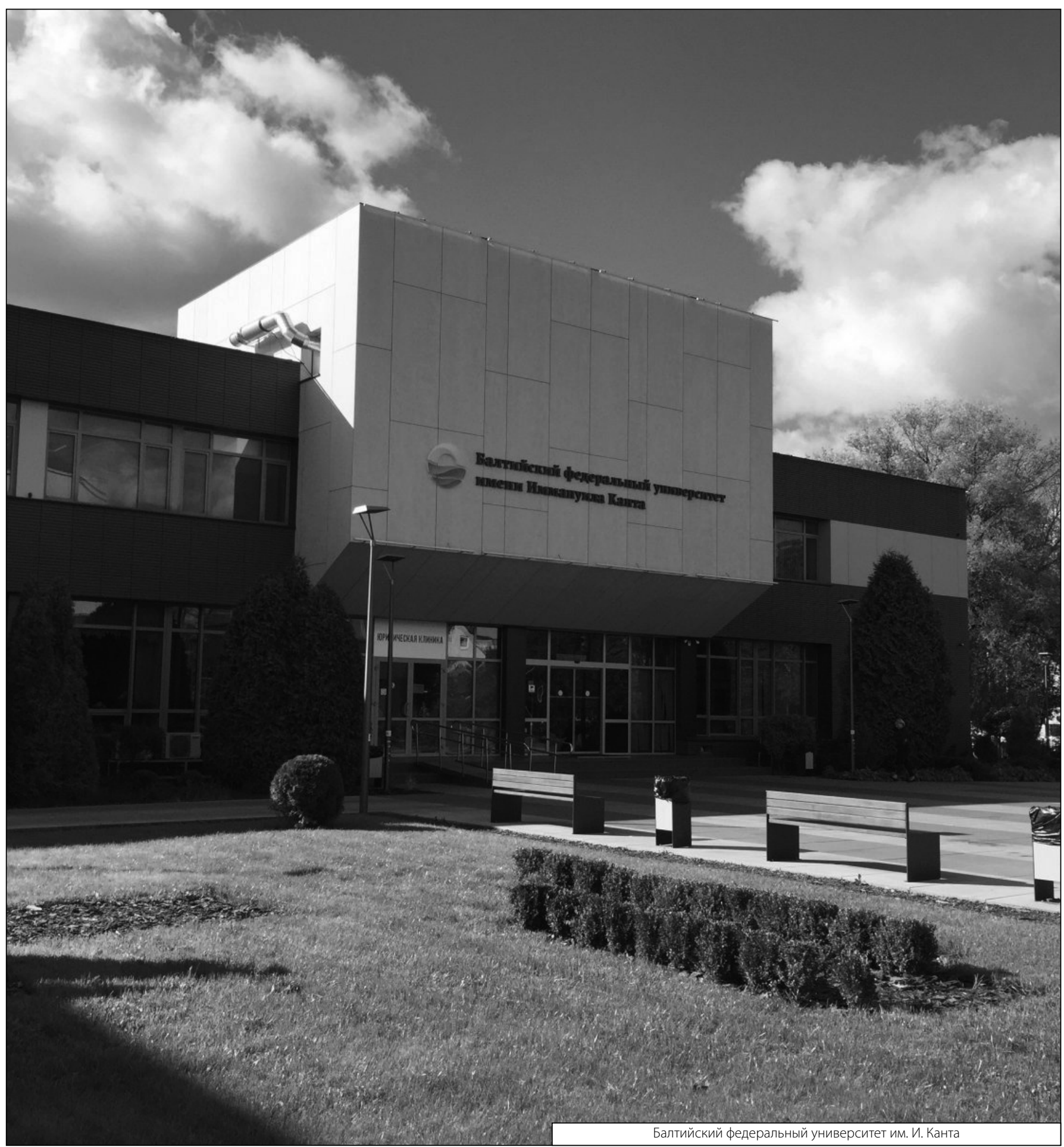

